\title{
A novel multiple criteria decision-making approach based on fuzzy DEMATEL, fuzzy ANP and fuzzy AHP for mapping collection and distribution centers in reverse logistics
}

\author{
Ocampo, L.A. ${ }^{a,}{ }^{,}$, Himang, C.M. ${ }^{b}$, Kumar, A. ${ }^{c}$, Brezocnik, M. $^{d}$ \\ ${ }^{a}$ Department of Industrial Engineering, Cebu Technological University, Cebu City, Philippines \\ ${ }^{\mathrm{b}}$ Graduate School, Cebu Technological University, Cebu City, Philippines \\ ${ }^{c}$ Centre for Supply Chain Improvement, University of Derby, United Kingdom \\ ${ }^{d}$ Faculty of Mechanical Engineering, Intelligent Manufacturing Systems Laboratory, University of Maribor, Maribor, Slovenia
}

\begin{abstract}
A B S T R A C T
The strategic location of reverse logistics facilities enables organizations to obtain optimal performance to collect end-of-line (EOL) products and distribute remanufactured products effectively and efficiently. The planning of facility location entails consideration of multiple essential criteria rather than optimizing a single criterion. This paper develops a methodological framework based on an integrated multiple criteria decision-making (MCDM) approach that captures the complexity of location planning for collection and distribution centers under fuzzy conditions utilizing decision making trial and evaluation laboratory (DEMATEL), analytic network process (ANP), and analytic hierarchy process (AHP). This novel approach aids decision-makers to simultaneously select a separate location for collection and distribution through a holistic assessment of a location's viability for both purposes. It advances the reverse logistics literature by considering multiple criteria and their interrelationships in the location selection process, along with uncertainty and vagueness in decision making. Additionally, the proposed approach allows flexibility for decision-makers as they retain the control in picking a site based on its priority on being a collection or distribution center. Results show that government policies and regulations play a vital role in the facility location decision as they interact mostly with other criteria. Moreover, results also suggest that quantity and quality uncertainties for remanufacturing are significant factors that must be taken into consideration in the collection function, while economic and market-oriented issues are major concerns for a distribution function. This finding was observed through the application of the proposed methodological framework in a case study of the furniture industry in the Philippines. The practical implications of this study focus on being an aid in organizing and improving the operations of the reverse logistics sector of the Philippines. Finally, the proposed approach can be used to address general facility location problems in other industrial applications where tradeoffs among stakeholders or entities are well pronounced and decision-makers find it imperative that such tradeoffs must be carefully considered.
\end{abstract}

(c) 2019 CPE, University of Maribor. All rights reserved.

\section{ARTICLE INFO}

Keywords:

Reverse logistics;

Collection;

Distribution;

Fuzzy environment;

Remanufacturing;

Multiple criteria decision-making (MCDM);

Decision-making and trial evaluation laboratory (DEMATEL);

Analytic network process (ANP);

Analytic hierarchy process (AHP)

${ }^{*}$ Corresponding author:

lanndonocampo@gmail.com

(Ocampo, L.A.)

Article history:

Received 13 November 2018

Revised 9 September 2019

Accepted 12 September 2019 


\section{References}

[1] Mutha, A., Pokharel, S. (2009). Strategic network design for reverse logistics and remanufacturing using new and old product modules, Computers \& Industrial Engineering, Vol. 56, No. 1, 334-346, doi: 10.1016/i.cie.2008. $\underline{06.006}$.

[2] Sheu, J.-B. (2011). Bargaining framework for competitive green supply chains under governmental financial intervention, Transportation Research Part E: Logistics and Transportation Review, Vol. 47, No. 5, 573-592, doi: 10.1016/j.tre.2010.12.006.

[3] Rashid, A., Asif, F.M.A., Krajnik, P., Nicolescu, C.N. (2013). Resource conservative manufacturing: An essential change in business and technology paradigm for sustainable manufacturing, Journal of Cleaner Production, Vol. 57, 166-177, doi: 10.1016/i.jclepro.2013.06.012.

[4] Govindan, K., Diabat, A., Shankar, K.M. (2015). Analyzing the drivers of green manufacturing with fuzzy approach, Journal of Cleaner Production, Vol. 96, 182-193, doi: 10.1016/i.jclepro.2014.02.054

[5] Govindan, K., Soleimani, H., Kannan, D. (2015). Reverse logistics and closed-loop supply chain: A comprehensive review to explore the future, European Journal of Operational Research, Vol. 240, No. 3, 603-626, doi: 10.1016/ j.ejor.2014.07.012.

[6] USITC (2012). Remanufactured goods: An overview of the U.S. and global industries, markets, and trade, United States International Trade Commission Publication, Investigation No. 332-525, USITC Publication 4356, Washington, USA.

[7] Wei, S., Cheng, D., Sundin, E., Tang, O. (2015). Motives and barriers of the remanufacturing industry in China, Journal of Cleaner Production, Vol. 94, 340-351, doi: 10.1016/i.jclepro.2015.02.014.

[8] Rathore, P., Kota, S., Chakrabarti, A. (2011). Sustainability through remanufacturing in India: A case study on mobile handsets, Journal of Cleaner Production, Vol. 19, No. 15, 1709-1722, doi: 10.1016/i.jclepro.2011.06.016.

[9] Chen, J.-M., Chang, C.-I. (2012). The co-operative strategy of a closed-loop supply chain with remanufacturing, Transportation Research Part E: Logistics and Transportation Review, Vol. 48, No. 2, 387-400, doi: 10.1016/i.tre. 2011.10.001.

[10] Xiaoyan, W. (2012). Research on design management based on green remanufacturing engineering, Systems Engineering Procedia, Vol. 4, 448-454, doi: 10.1016/i.sepro.2012.01.009.

[11] Srivastava, S.K. (2006). Network design for reverse logistics, Omega, Vol. 36, No. 4, 535-548, doi: 10.1016/i. omega.2006.11.012.

[12] Nnorom, I.C., Osibanjo, O. (2008). Overview of electronic waste (e-waste) management practices and legislations, and their poor applications in the developing countries, Resources, Conservation, and Recycling, Vol. 52, No. 6, 843-858, doi: 10.1016/i.resconrec.2008.01.004.

[13] Zou, Z.-B., Wang, J.-J., Deng, G.-S., Chen, H. (2016). Third-party remanufacturing mode selection: Outsourcing or authorization?, Transportation Research Part E: Logistics and Transportation Review, Vol. 87, 1-19, doi: 10.1016/ j.tre.2015.12.008.

[14] Malik, S., Kumari, A., Agrawal, S. (2015). Selection of locations of collection centers for reverse logistics using GTMA, Materials Today: Proceedings, Vol. 2, No. 4-5, 2538-2547, doi: 10.1016/i.matpr.2015.07.199.

[15] Pop, P.C., Pinteaa, C.-M., Pop Sitar, C., Hajdu-Măcelaru, M. (2015). An efficient reverse distribution system for solving sustainable supply chain network design problem, Journal of Applied Logic, Vol. 13, No. 2, Part A, 105113, doi: 10.1016/i.jal.2014.11.004.

[16] Hong, I.-H., Yeh, J.-S. (2012). Modeling closed-loop supply chains in the electronics industry: A retailer collection application, Transportation Research Part E: Logistics and Transportation Review, Vol. 48, No. 4, 817-829, doi: 10.1016/i.tre.2012.01.006

[17] Atasu, A., Toktay, L.B., Van Wassenhove, L.N. (2013). How collection cost structure drives the manufacturer's reverse channel choice, Production and Operations Management, Vol. 22, No. 5, 1089-1102, doi: 10.1111/i.19375956.2012.01426.x.

[18] Wojanowski, R., Verter, V., Boyaci, T. (2007). Retail-collection network design under deposit-refund, Computers \& Operations Research, Vol. 34, No. 2, 324-345, doi: 10.1016/i.cor.2005.03.003.

[19] Min, H., Ko, H.-J. (2008). The dynamic design of a reverse logistics network from the perspective of third-party logistics service providers, International Journal of Production Economics, Vol. 113, No. 1, 176-192, doi: 10.1016 Li.ijpe.2007.01.017.

[20] Paksoy, T., Pehlivan, N.Y., Kahraman, C. (2012). Organizational strategy development in distribution channel management using fuzzy AHP and hierarchical fuzzy TOPSIS, Expert Systems with Applications, Vol. 39, No. 3 , 2822-2841, doi: 10.1016/i.eswa.2011.08.142.

[21] Govindan, K., Chaudhuri, A. (2016). Interrelationships of risks faced by third party logistics service providers: A DEMATEL based approach, Transportation Research Part E: Logistics and Transportation Review, Vol. 90, 177195, doi: 10.1016/i.tre.2015.11.010.

[22] Chiou, C.Y., Chen, H.C, Yu, C.T., Yeh, C.Y. (2012). Consideration factors of reverse logistics implementation - A case study of Taiwan's electronics industry, Procedia - Social and Behavioral Sciences, Vol. 40, 375-381, doi 10.1016/i.sbspro.2012.03.203.

[23] Zadeh, L.A. (1965). Fuzzy sets, Information and Control, Vol. 8, No. 3, 338-353, doi: 10.1016/S0019-9958(65)90 241-X.

[24] Serrano, C., Aggoune-Mtalaa, W., Sauer, N. (2013). Dynamic models for green logistic networks design, IFAC Proceedings Volumes, Vol. 46, No. 9, 736-741, doi: 10.3182/20130619-3-RU-3018.00101. 
[25] Langevin, A., Mbaraga, P., Campbell, J.F. (1996). Continuous approximation models in freight distribution: An overview, Transportation Research Part B: Methodological, Vol. 30, No. 3, 163-188, doi: 10.1016/0191-2615(95) $\underline{00035-6 .}$

[26] Yang, X. (2013). A review of distribution related problems in logistics and supply chain research, International Journal of Supply Chain Management, Vol. 2, No. 4, 1-8.

[27] Mukherjee, K., Mondal, S. (2009). Analysis of issues relating to remanufacturing technology - A case of an Indian company, Technology Analysis \& Strategic Management, Vol. 21, No. 5, 639-652, doi: 10.1080/0953732090296 $\underline{9174 .}$

[28] Choudhary, N., Singh, N.K. (2011). Remanufacturing in India: Approaches, potentials \& technical challenges, International Journal of Industrial Engineering and Technology, Vol. 3, No. 3, 223-227.

[29] Sharma, V., Garg, S.K., Sharma, P.B. (2016). Identification of major drivers and roadblocks for remanufacturing in India, Journal of Cleaner Production, Vol. 112, Part 3, 1882-1892, doi: 10.1016/i.jclepro.2014.11.082.

[30] Yi, P., Huang, M., Guo, L., Shi, T. (2016). A retailer oriented closed-loop supply chain network design for end of life construction machinery remanufacturing, Journal of Cleaner Production, Vol. 124, 191-203, doi: 10.1016/ j.jclepro.2016.02.070.

[31] Owen, S.H, Daskin, M.S. (1998). Strategic facility location: A review, European Journal of Operational Research, Vol. 111, No. 3, 423-447, doi: 10.1016/S0377-2217(98)00186-6.

[32] Zhang, Y., Snyder, L.V., Ralphs, T.K., Xue, Z. (2016). The competitive facility location problem under disruption risks, Transportation Research Part E: Logistics and Transportation Review, Vol. 93, 453-473, doi: 10.1016/j.tre. 2016.07.002.

[33] Kuo, M.-S. (2011). Optimal location selection for an international distribution center by using a new hybrid method, Expert Systems with Applications, Vol. 38, No. 6, 7208-7221, doi: 10.1016/i.eswa.2010.12.002.

[34] Sarkis, J., Gonzalez-Torre, P., Adenso-Diaz, B. (2010). Stakeholder pressure and the adoption of environmental practices: The mediating effect of training, Journal of Operations Management, Vol. 28, No. 2, 163-176, doi: 10.1016/i.jom.2009.10.001.

[35] Zhang, T., Chu, J., Wang, X., Liu, X., Cui, P. (2011). Development pattern and enhancing system of automotive components remanufacturing industry in China, Resources, Conservation, and Recycling, Vol. 55, No. 6, 613-622, doi: 10.1016/i.resconrec.2010.09.015.

[36] Nunes, K.R.A., Mahler, C.F., Valle, R.A. (2009). Reverse logistics in the Brazilian construction industry, Journal of Environmental Management, Vol. 90, No. 12, 3717-3720, doi: 10.1016/i.jenvman.2008.05.026.

[37] Govindan, K., Khodaverdi, R., Vafadarnikjoo, A. (2016). A grey DEMATEL approach to develop third-party logistics provider selection criteria, Industrial Management \& Data Systems, Vol. 116, No. 4, 690-722, doi: 10.1108/ IMDS-05-2015-0180.

[38] Govindan, K., Madan Shankar, K., Kannan, D. (2016). Application of fuzzy analytic network process for barrier evaluation in automotive parts remanufacturing towards cleaner production: A study in an Indian scenario, Journal of Cleaner Production, Vol. 114, 199-213, doi: 10.1016/i.jclepro.2015.06.092.

[39] Ijomah, W.L., McMahon, C.A., Hammond, G.P., Newman, S.T. (2007). Development of design for remanufacturing guidelines to support sustainable manufacturing, Robotics and Computer-Integrated Manufacturing, Vol. 23, No. 6, 712-719, doi: 10.1016/i.rcim.2007.02.017.

[40] Xu, L., Mathiayazhagan, K., Govindan, K., Noorol Haq, A., Ramachandran, N.V., Ashokkumar, A. (2013). Multiple comparative studies of green supply chain management: Pressures analysis, Resources, Conservation and Recycling, Vol. 78, 26-35, doi: 10.1016/i.resconrec.2013.05.005.

[41] Saavedra, Y.M.B., Barquet, A.P.B., Rozenfeld, H., Forcellini, F.A., Ometto, A.R. (2013). Remanufacturing in Brazil: Case studies on the automotive sector, Journal of Cleaner Production, Vol. 53, 267-276, doi: 10.1016/i.jclepro. 2013.03.038.

[42] Promentilla, M.A.B., Furuichi, T., Ishii, K., Tanikawa, N. (2008). A fuzzy analytic network process for multicriteria evaluation of contaminated site remedial countermeasures, Journal of Environmental Management, Vol. 88, No. 3, 479-495, doi: 10.1016/i.jenvman.2007.03.013.

[43] Tseng, M.-L. (2011). Using a hybrid MCDM model to evaluate firm environmental knowledge management in uncertainty, Applied Soft Computing, Vol. 11, No. 1, 1340-1352, doi: 10.1016/i.asoc.2010.04.006.

[44] Tseng, M.L., Lin, Y.H., Chiu, A.S.F., Liao, J.C.H. (2008). Using FANP approach on selection of competitive priorities based on cleaner production implementation: A case study in PCB manufacturer, Taiwan, Clean Technologies and Environmental Policy, Vol. 10, No. 1, 17-29, doi: 10.1007/s10098-007-0109-4.

[45] Fontela, E., Gabus, A. (1972). World problems an invitation to further thought within the framework of DEMATEL, Battelle Geneva Research Centre, Geneva.

[46] Gabus, A., Fontela, E. (1973). Perceptions of the world problem atique: Communication procedure, communicating with those bearing collective responsibility, DEMATEL Report No.1, Battelle Geneva Research Centre, Geneva.

[47] Wang, R.-C., Chuu, S.-J. (2004). Group decision-making using a fuzzy linguistic approach for evaluating the flexibility in a manufacturing system, European Journal of Operational Research, Vol. 154, No. 3, 563-572, doi: 10.1016/S0377-2217(02) 00729-4.

[48] Tsai, W.-H., Chou, W.-C. (2009). Selecting management systems for sustainable development in SMEs: A novel hybrid model based on DEMATEL, ANP, and ZOGP, Expert Systems with Applications, Vol. 36, No. 2, Part 1, $1444-$ 1458, doi: 10.1016/i.eswa.2007.11.058.

[49] Büyüközkan, G., Çifçi, G. (2012). A novel hybrid MCDM approach based on fuzzy DEMATEL, fuzzy ANP and fuzzy TOPSIS to evaluate green suppliers, Expert Systems with Applications, Vol. 39, No. 3, 3000-3011, doi: 10.1016/ j.eswa.2011.08.162. 
[50] Bongo, M.F., Ocampo, L.A. (2017). A hybrid fuzzy MCDM approach for mitigating airport congestion: A case in Ninoy Aquino international airport, Journal of Air Transport Management, Vol. 63, 1-16, doi: 10.1016/ j.jairtraman.2017.05.004.

[51] Lin, R.-J. (2013). Using fuzzy DEMATEL to evaluate the green supply chain management practices, Journal of Cleaner Production, Vol. 40, 32-39, doi: 10.1016/i.jclepro.2011.06.010.

[52] Baykasoğlu, A., Kaplanoğlu, V., Durmuşoğlu, Z.D.U., Şahin, C. (2013). Integrating fuzzy DEMATEL and fuzzy hierarchical TOPSIS methods for truck selection, Expert Systems with Applications, Vol. 40, No. 3, 899-907, doi: 10.1016/i.eswa.2012.05.046.

[53] Kumar, A., Mussada, E.K., Ashif, M., Tyagi, D., Srivastava, A.K. (2017). Fuzzy Delphi and hybrid AH-MATEL integration for monitoring of paint utilization, Advances in Production Engineering \& Management, Vol. 12, No. 1, 4150, doi: 10.14743/apem2017.1.238.

[54] Saaty, T.L. (1977). A scaling method for priorities in hierarchical structures, Journal of Mathematical Psychology, Vol. 15, No. 3, 234-281, doi: 10.1016/0022-2496(77)90033-5.

[55] Saaty, T.L. (1996). Decision making with dependence and feedback: The analytic network process, RWS Publications, Pittsburgh, USA.

[56] Shyur, H.-J. (2006). COTS evaluation using modified TOPSIS and ANP, Applied Mathematics and Computation, Vol. 177, No. 1, 251-259, doi: 10.1016/i.amc.2005.11.006.

[57] Tavana, M., Khalili-Damghani, K., Abtahi, A.-R. (2013). A hybrid fuzzy group decision support framework for advanced-technology prioritization at NASA, Expert Systems with Applications, Vol. 40, No. 2, 480-491, doi: 10.1016/i.eswa.2012.07.040.

[58] Chen, S.H., Wang, P.W., Chen, C.M., Lee, H.T. (2010). An analytic hierarchy process approach with linguistic variables for selection of an R\&D strategic alliance partner, Computers \& Industrial Engineering, Vol. 58, No. 2, 278287, doi: 10.1016/i.cie.2009.10.006.

[59] Liao, C.-N. (2011). Fuzzy analytical hierarchy process and multi-segment goal programming applied to new product segmented under price strategy, Computers \& Industrial Engineering, Vol. 61, No. 3, 831-841, doi: 10.1016/i.cie.2011.05.016.

[60] Wu, C., Zhang, X.-Y., Yeh, I.-C., Chen, F.-Y., Bender, J., Wang, T.-N. (2013). Evaluating competitiveness using fuzzy analytic hierarchy process - A case study of Chinese airlines, Journal of Advanced Transportation, Vol. 47, No. 7, 619-634, doi: 10.1002/atr.183.

[61] Wang, Y., Jung, K.-A., Yeo, G.-T., Chou, C.-C. (2014). Selecting a cruise port of call location using the fuzzy-AHP method: A case study in East Asia, Tourism Management, Vol. 42, 262-270, doi: 10.1016/j.tourman.2013.11.005.

[62] Dožić, S., Lutovac, T., Kalić, M. (2017). Fuzzy AHP approach to passenger aircraft type selection, Journal of Air Transport Management, Vol. 68, 165-175, doi: 10.1016/i.jairtraman.2017.08.003.

[63] Banduka, N., Tadić, D., Mačužić, I., Crnjac, M. (2018). Extended process failure mode and effect analysis (PFMEA) for the automotive industry: The FSQC-PFMEA, Advances in Production Engineering \& Management, Vol. 13, No. 2, 206-215, doi: 10.14743/apem 2018.2.285.

[64] Razmi, J., Rafiei, H., Hashemi, M. (2009). Designing a decision support system to evaluate and select suppliers using fuzzy analytic network process, Computers \& Industrial Engineering, Vol. 57, No. 4, 1282-1290, doi: 10.1016/i.cie.2009.06.008.

[65] Ayağ, Z., Özdemir, R.G. (2009). A hybrid approach to concept selection through fuzzy analytic network process, Computers \& Industrial Engineering, Vol. 56, No. 1, 368-379, doi: 10.1016/i.cie.2008.06.011.

[66] Babaesmailli, M., Arbabshirani, B., Golmah, V. (2012). Integrating analytical network process and fuzzy logic to prioritize the strategies - A case study for tile manufacturing firm, Expert Systems with Applications, Vol. 39, No. 1, 925-935, doi: 10.1016/i.eswa.2011.07.090.

[67] Uygun, Ö., Kaçamak, H., Kahraman, Ü.A. (2014). An integrated DEMATEL and Fuzzy ANP techniques for evaluation and selection of outsourcing provider for a telecommunication company, Computers \& Industrial Engineering, Vol. 86, 137-146, doi: 10.1016/i.cie.2014.09.014.

[68] Lin, C.-L., Wu, W.-W. (2004). A fuzzy extension of the DEMATEL method for group decision making, European Journal of Operational Research, Vol. 156, 445-455.

[69] Ocampo, L., Clark, E., Tanudtanud, K.V. (2015). Structural decisions of sustainable manufacturing strategy with fuzzy analytic network process (FANP), International Journal of Strategic Decision Sciences, Vol. 6, No. 2, 12-27, doi: 10.4018/ijsds.2015040102.

[70] Saaty, T.L. (2008). Decision making with the analytic hierarchy process, International Journal Services Sciences, Vol. 1, No. 1, 83-98, doi: 10.1504/IJSSCI. 2008.017590.

[71] Mikhailov, L., Tsvetinov, P. (2004). Evaluation of services using a fuzzy analytic hierarchy process, Applied Soft Computing, Vol. 5, No. 1, 23-33, doi: 10.1016/i.asoc.2004.04.001.

[72] Wang, Y.-M., Chin, K.-S. (2011). Fuzzy analytic hierarchy process: A logarithmic fuzzy preference programming methodology, International Journal of Approximate Reasoning, Vol. 52, No. 4, 541-553, doi: 10.1016/i.ijar.2010. $\underline{12.004}$.

[73] Krishnan, T.N., Poulose, S. (2016). Response rate in industrial surveys conducted in India: Trends and implications, IIMB Management Review, Vol. 28, No. 2, 88-97, doi: 10.1016/i.iimb.2016.05.001.

[74] Kannan, G. (2009). Fuzzy approach for the selection of third party reverse logistics provider, Asia Pacific Journal of Marketing and Logistics, Vol. 21, No. 3, 397-416, doi: 10.1108/13555850910973865.

[75] Govindan, K., Pokharel, S., Kumar, P.S. (2009). A hybrid approach using ISM and fuzzy TOPSIS for the selection of reverse logistics provider, Resources, Conservation and Recycling, Vol. 54, No. 1, 28-36, doi: 10.1016/i.resconrec. 2009.06.004. 
[76] Mittal, V.K., Sangwan, K.S. (2014). Prioritizing barriers to green manufacturing: Environmental, social and economic perspectives, Procedia CIRP, Vol. 17, 559-564, doi: 10.1016/i.procir.2014.01.075.

[77] Ocampo, L.A., Promentilla, M.A.B. (2016). Development of a sustainable manufacturing strategy using analytic network process, International Journal of Business and Systems Research, Vol. 10, No. 2-4, 262-290, doi: 10.1504 LIJBSR.2016.075744.

[78] Xiang, W., Ming, C. (2011). Implementing extended producer responsibility: Vehicle remanufacturing in China, Journal of Cleaner Production, Vol. 19, No. 6-7, 680-686, doi: 10.1016/i.jclepro.2010.11.016.

[79] Andel, T., Aichlmayr, M. (2002). Turning returns into cash, Transportation Distribution, Vol. 43, No. 8, 29-39.

[80] McAllister, J. (2015). Factors influencing solid-waste management in the developing world, All Graduate Plan B and other Reports, Utah State University, Logan, Utah, USA.

[81] Magtolis, C.M., Indab, J.D. (2008). A policy study on environmental protection in the Philippines, Philippine Journal of Public Administration, Vol. 52, No. 2-4, 364-379.

[82] Toffel, M.W. (2004). Strategic management of product recovery, California Management Review, Vol. 46, No. 2, 120-141, doi: $10.2307 / 41166214$.

[83] Aras, N., Aksen, D., Tanuğur, A.G. (2008). Locating collection centres for incentive-dependent returns under a pick-up policy with capacitated vehicles, European Journal of Operational Research, Vol. 191, No. 3, 1223-1240, doi: $10.1016 /$ i.ejor.2007.08.002.

[84] Rao, C., Goh, M., Zhao, Y., Zheng, J. (2015). Location selection of city logistics centers under sustainability, Transportation Research Part D: Transport and Environment, Vol. 36, 29-44, doi: 10.1016/i.trd.2015.02.008.

[85] Mitra, S. (2007). Revenue management for remanufactured products, Omega, Vol. 35, No. 5, 553-562, doi: 10.1016/i.omega.2005.10.003

[86] Govindan, K., Palaniappan, M., Zhu, Q., Kannan, D. (2012). Analysis of third party reverse logistics provider using interpretive structural modeling, International Journal of Production Economics, Vol. 140, No. 1, 204-211, doi: 10.1016/j.ijpe.2012.01.043.

[87] PwC Cebu 2017 CEO Survey (2017), from https://www.pwc.com/ph/en/ceo-survey/2017/great-expectationscebu-ceo-survey-2017.pdf, accessed November 13, 2018.

[88] DTI BOI (2016). Securing the Future of Philippine industries: Furniture, from http://industry.gov.ph/industry/ furniture/ accessed November 13, 2018. 


\title{
Nov pristop odločanja z več kriteriji, ki temelji na mehki metodi DEMATEL, mehkem ANP in mehkem AHP za lociranje zbirnih in distribucijskih centrov $\mathbf{v}$ vzvratni logistiki
}

\author{
Ocampo, L.A. ${ }^{a,}{ }^{*}$, Himang, C.M. ${ }^{b},{ }^{\prime}$ Kumar, A. ${ }^{c}$, Brezocnik, M. $^{d}$ \\ ${ }^{a}$ Department of Industrial Engineering, Cebu Technological University, Cebu City, Philippines \\ ${ }^{\mathrm{b}}$ Graduate School, Cebu Technological University, Cebu City, Philippines \\ ${ }^{\mathrm{c} C}$ Centre for Supply Chain Improvement, University of Derby, United Kingdom \\ ${ }^{d}$ Faculty of Mechanical Engineering, Intelligent Manufacturing Systems Laboratory, University of Maribor, Maribor, Slovenia
}

\section{POVZETEK}

Strateška lokacija objektov vzvratne logistike omogoča organizacijam, da pridobijo optimalno zmogljivost za zbiranje odsluženih izdelkov (EOL) in učinkovito distribucijo obnovljenih izdelkov. Načrtovanje lokacije objekta vključuje upoštevanje več bistvenih meril in ne optimizacije enega samega merila. $V$ tem prispevku je razvit metodološki okvir, ki temelji na integriranem pristopu odločanja z več kriteriji (MCDM), ki zajema kompleksnost izbire lokacije za zbirne in distribucijske centre pod mehkimi pogoji $\mathrm{z}$ uporabo metode DEMATEL, procesa analitične mreže (ANP) in analitičnega hierarhičnega procesa (AHP). Ta nov pristop pomaga odločevalcem, da hkrati izberejo ločeno lokacijo za zbiranje in distribucijo s celostno oceno sposobnosti preživetja lokacije za oba cilja. Nadgrajuje se znanje o vzvratni logistiki z upoštevanjem več meril in medsebojne povezanosti v postopku izbire lokacije, skupaj z negotovostjo in nejasnostjo pri sprejemanju odločitev. Poleg tega predlagani pristop omogoča odločevalcem prilagodljivost, saj obdržijo nadzor pri izbiri mesta glede na prednostno nalogo zbiranja ali distribucije. Rezultati kažejo, da imajo vladne politike in predpisi ključno vlogo pri odločitvi o lokaciji objekta, saj večinoma vplivajo na druga merila. Poleg tega rezultati kažejo tudi na to, da so količinske in kakovostne negotovosti za obnovo izdelka pomembni dejavniki, ki jih je treba upoštevati pri zbiranju, medtem ko so ekonomska in tržno usmerjena vprašanja glavna skrb za distribucijsko funkcijo. To je bilo ugotovljeno z uporabo predlaganega metodološkega okvira v študiji primera pohištvene industrije na Filipinih. Praktične posledice te študije so usmerjene v pomoč pri organizaciji in izboljšanju poslovanja Filipinov z vzvratno logistiko. Predlagani pristop je mogoče uporabiti za reševanje splošnih težav z lokacijo objektov tudi v drugih industrijskih aplikacijah, kjer so kompromisi med zainteresiranimi stranmi ali subjekti dobro izraženi in odločevalci menijo, da je treba takšne kompromise skrbno upoštevati.

\section{PODATKI O ČLANKU}

Ključne besede:

Vzvratna logistika;

Zbiranje;

Distribucija;

Mehko okolje;

Predelava;

Odločanje z več kriteriji (MCDM);

Metoda DEMATEL;

Analitični mrežni proces (ANP);

Analitični hierarhični proces (AHP)

*Kontaktna oseba:

lanndonocampo@gmail.com

(Ocampo, L.A.)

Zgodovina članka:

Prejet 13. novembra 2018

Popravljen 9. septembra 2019

Sprejet 12. septembra 2019 